\title{
Automatic Analysis of the Elastic Fiber Texture of the Aorta
}

\author{
G. Vieira ${ }^{1}$, R. L Adam², D. P. Ferro ${ }^{1}$, A. A. de Thomaz ${ }^{3}$, C. L. Cesar ${ }^{3}$, and K. Metze ${ }^{1}$ \\ 1. Faculdade de Ciências Médicas, Universidade Estadual de Campinas ( UNICAMP), Brazil \\ 2. Instituto de Computação, Universidade Estadual de Campinas ( UNICAMP), Brazil \\ 3. CePOF, Instituto de Física, Universidade Estadual de Campinas, Brazil
}

The elastic fiber network is an essential component of arteries, especially of the aorta. Loss or remodeling of the elastic fiber texture accompanies many diseases of the vessels and may provoke life-threatening events, such as dissection or rupture. The study of these phenomena is important for the understanding of the pathophysiology of these events. Classical geometric analysis cannot describe adequately texture details of the elastic fiber network, such as waviness, folding or orientation of the fibers. Therefore we created a program which enables us to characterize, in an objective manner, texture alterations of the elastic structures. Elastic fibers can be easily demonstrated in eosin-stained histologic sections examined by fluorescence microscopy. Therefore we used computerized analysis of large confocal fluorescence images. For texture analysis we applied features of the fractal dimensions, the gray-level co-occurence matrix, and characteristics derived from Fast-Fourier-transformed (FFT) images. The confocal system was composed by the Olympus IX-81 inverted microscope, the FV300 scan head and the FV-5 COMB2 laser combiner. We used two Hamamatsu model 3896 PMTs, one PMT3 to collect the transmitted laser light to acquire the transmission image and the other, PMT1 to collect confocal backscattered fluorescence. The fluorescence of the eosin-stained slides was excited with the $488 \mathrm{~nm}$ line of an Argon laser [Omnichrome, Model 170B] coupled to the scan head through an optical fiber. The whole fluorescence band was collect without any filter in front of the PMTs. The FV300 dichroic mirror suppresses any back-reflected argon laser light. Each $220 \square \mathrm{m}$ x $220 \square \mathrm{m}$ image was acquired with 512 x 512 pixels resolution using a 40x 1.3 NA oil immersion (UPlanFL N Olympus) objective. This means that each pixel has the $430 \mathrm{~nm}$ x $430 \mathrm{~nm}$ dimensions. The Fluoview software was used to reconstruct the images. The full image of the whole sample ( 2 or $3 \mathrm{~mm}$ long) was composed by several $220 \mu \mathrm{m}$ frames. After each frame a Prior Scientific, model ProScan translation stage was used to move the specimens in the $x-y$ direction to acquire the next frame. The procedure to maintain the elastin fibers aligned vertically was the following: after finding the first region of interest we digitally rotate the acquisition area until the fibers were aligned vertically and obtained the angle $\theta$ reading from the computer screen. The subsequent translation for the next frame then followed the rule $(\mathrm{x}$ or $\mathrm{y})$ translation $=210(\cos \theta$ or $\sin \theta$ in microns. The translation of $210 \mu \mathrm{m}$ assured an overlapping between subsequent frames to be later used to merge the images to a large unique image representing the whole region of interest. Then a gliding box of $128 \times 128$ pixels was running in 1-pixel steps along a predefined axis parallel to the bottom line of the image. For every new box, texture features (variables derived from the concurrence matrix, energy, entropies, fractal dimensions etc) were calculated and plotted in diagrams, where the position on the x-axis was equivalent to the topography in the histologic picture. A more detailed representation of the wavines of the fibers was achieved by an analysis of different sectors of the FFT transformed images. Figure 1 illustrates this method in a section from a human aorta, showing the correspondence between the histologic fluorescence image and diagram, thus emphasizing the local architectural disturbances. In conclusion we show that the presented method provides a new tool for an objective and reproducible automatic texture analysis of the elastic fiber texture of the aorta with the possibility of quantification of the disease process. 


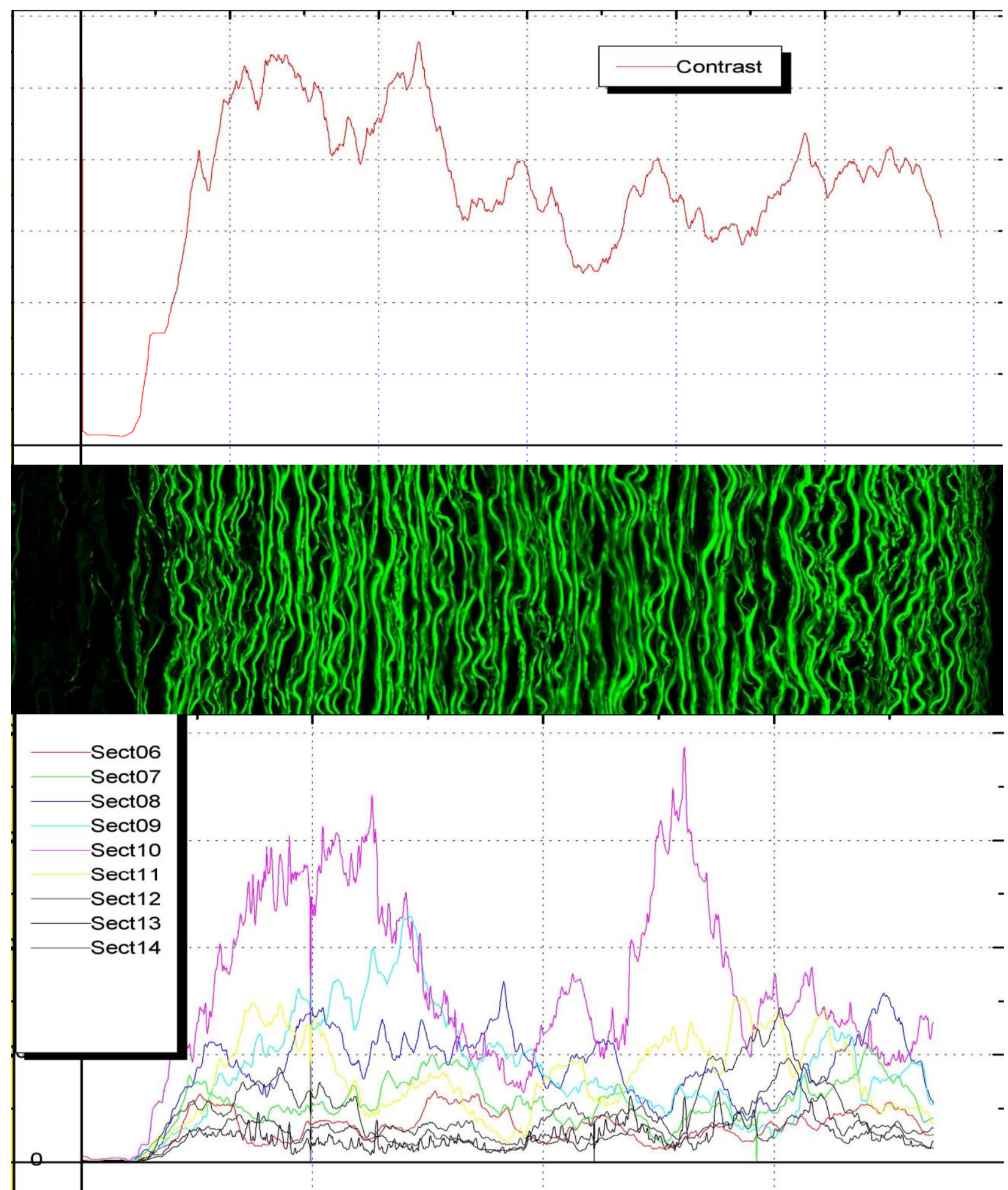

Figure 1. Upper part: diagram showing the co-occurrence matrix derived variable "contrast" of the gliding-boxes. Central part: haematoxylin-eosin stained fluorescence image of a human aorta. Lower part: corresponding diagram of the energy values of different sectors in the FFT transformed images of the gliding boxes. Sector 6 is equivavent to an inclination between 60 and 70 degrees from the $\mathrm{x}$ axis. Sector 7 represents 70 to 80 degrees and so on. In this example sector 10 detects best the architectural disturbances in the aorta in the right part of the fluorescence picture. 